\title{
HDUST3 - A chemically realistic, 3-D, NLTE radiative transfer code
}

\author{
Alex C. Carciofi ${ }^{1}$, Jon E. Bjorkman ${ }^{2}$ and Janos Zsargó ${ }^{3}$ \\ ${ }^{1}$ Instituto de Astronomia, Geofísica e Ciências Atmosféricas, Universidade de São Paulo, \\ Rua do Matão 1226, Cidade Universitária, 05508-900 São Paulo, SP, Brazil \\ email: carciofi@usp.br \\ ${ }^{2}$ Ritter Observatory, Dept. of Phys. \& Astron., MS 113, Univ. of Toledo, Toledo, OH 43606 \\ ${ }^{3}$ Escuela Superior de Física y Matemática. Instituto Politécnico Nacional. Av. Instituto \\ Politécnico Nacional, Edificio 9, C.P. 07738, DF. México
}

\begin{abstract}
HDUST is a 3D, NLTE radiative transfer code based on the Monte Carlo method. We report on recent advancements on the code, which is now capable of handling He and other elements in the NLTE regime and in 3D configurations. In this contribution we show initial comparisons with CMFGEN, made with spherical wind models composed of $\mathrm{H}+\mathrm{He}$.
\end{abstract}

Keywords. methods: numerical, radiative transfer

The HDUST code (Carciofi \& Bjorkman 2006; Carciofi et al. 2004) has been used already in over 30 refereed publications, mostly for modeling Be star disks, but also other systems such as hot star winds and dusty envelopes around hot and cool stars. The current stable version includes $\mathrm{H}$ in full NLTE for 3D geometries, as well as dust and free electrons.

We present the new version of the code (HDUST3), modified to include a general atomic module, that allows for the inclusion of other chemical elements. An inicial comparison is shown in Fig. 1 for a model with $R_{\star}=6.6 R_{\text {sun }}, T_{\text {eff }}=22000 \mathrm{~K}, \log g=4, \dot{M}=$ $2 \times 10^{-10} M_{\text {sun } \mathrm{yr}^{-1}}$, and $v_{\infty}=1600 \mathrm{kms}^{-1}$. The match for H I is quite good, but some discrepancies are found for HeI, which are likely due to the fact that the inner boundary condition (namely the photospheric spectrum) is not the same in the two simulations.
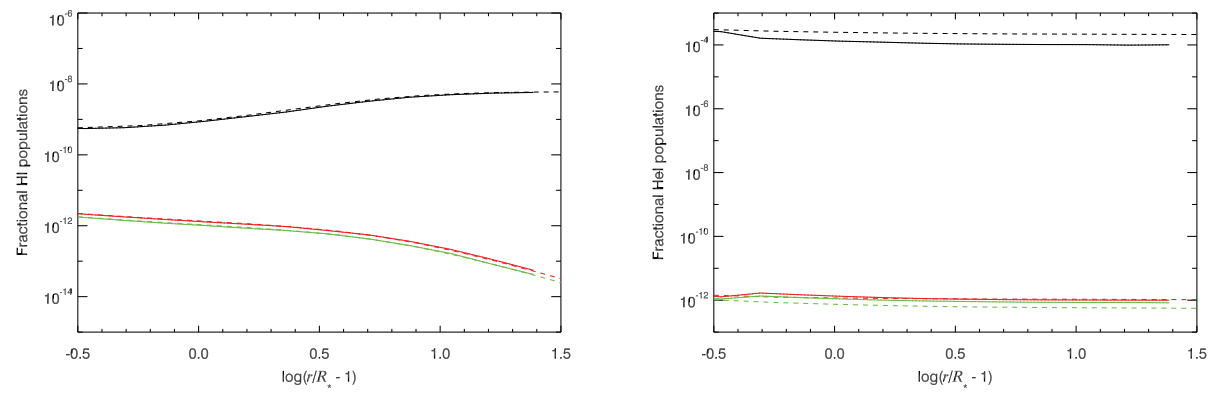

Figure 1. Fractional level populations vs. distance from the star $\left(n_{\text {level }} / n_{\text {element }}\right)$. Black: $n_{1}$. Red: $n_{2}$. Green: $n_{3}$. Solid line: HDUST. Dashed line: CMFGEN. Left: H I. Right: HeI.

\section{References}

Carciofi, A. C., Bjorkman, J. E., \& Magalhães, A. M. 2004, ApJ, 604, 238

Carciofi, A. C., \& Bjorkman, J. E. 2006, ApJ, 639, 1081 